International Journal of Pure and Applied Mathematics

Volume 115 No. $3 \quad 2017,539-548$

ISSN: 1311-8080 (printed version); ISSN: 1314-3395 (on-line version)

url: http://www.ijpam.eu

doi: 10.12732 /ijpam.v115i3.8

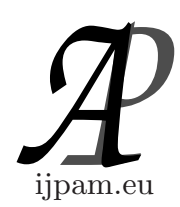

\title{
AN APPROPRIATE METHOD TO HANDLE FUZZY INTEGRO-DIFFERENTIAL EQUATIONS
}

\author{
S.P. Sathiyapriya ${ }^{1}$, S. Narayanamoorthy ${ }^{2}$ \\ ${ }^{1,2}$ Department of Mathematics \\ Bharathiar University \\ Coimbatore, 641046, Tamil Nadu, INDIA
}

\begin{abstract}
Several mathematical formulations of physical phenomena under uncertainty contain fuzzy integro-differential equations and due to their frequent appearance in several applied fields, an appropriate method for handling them is presented. Our proposed algorithm is given in detail and it can be treated as the extended form of homotopy perturbation method. We use an accelerating parameter to enhance the rate of convergence of the solution and construction of suitable homotopy facilitates the calculation of approximate solution in the form of convergent series with simple computable components.
\end{abstract}

AMS Subject Classification: 03B52, 45B02

Key Words: fuzzy integro-differential equation, homotopy perturbation method, accelerating parameter, approximate solution

\section{Introduction}

Many real world problems are described by fuzzy integral and fuzzy integrodifferential equations, which are helpful in studying the observability of dynamical control systems. Also various initial and boundary value problems of ordinary differential equations and partial differential equations are associated with them. This is the main reason to study these equations extensively. In

$\begin{array}{lr}\text { Received: } & \text { April 29,2017 } \\ \text { Revised: } & \text { June 20,2017 } \\ \text { Published: } & \text { July 27, } 2017 \\ \text { Correspondence } & \text { author }\end{array}$

(c) 2017 Academic Publications, Ltd. url: www.acadpubl.eu

${ }^{\S}$ Correspondence author 
fact, obtaining the exact solutions of such equations is not possible in all cases because of inherited restrictions form application of fuzzy concepts in these problems. In order to model the dynamical systems under uncertainty, we need to use the idea of fuzzy sets which was introduced by Zadeh in [11]. Later Dubois and Prade in [2] presented an elementary fuzzy calculus based on the extension principle. Alternative approaches were suggested by Goetschel and Voxman in [4], Kaleva in [7] and others. The fuzzy integral equations and fuzzy differential equations are the most important fields of fuzzy set theory. In recent years various authors have been focusing on the advanced and efficient methods for solving fuzzy intergo-differential equations and therefore investigating solution of them with high accuracy is of center of interest to many researchers. Among these techniques, homotopy perturbation method (HPM) is the most transparent methods of solution of them because it provide immediate as well as convergent series and in most cases only few iterations leads to highly accurate solutions. The HPM proposed by He (see [5], [6]) for solving integro-diferential equations (see [3], [9], [10]), linear and nonlinear has been the subject of extensive analytical and numerical studies. The method is a coupling of the traditional perturbation method and homotopy in topology which deforms to a simple problem that can be easily solved. Our proposed method can be considered as an extended form of HPM which uses an accelerating parameter to enhance the rate of convergence of solutions of linear fuzzy Fredholm integro-differential equation (FFIDE) of the form

$$
\widetilde{F}^{\prime}=\widetilde{q}(x) \widetilde{F}(x)+\lambda \int_{a}^{b} k(x, t) \widetilde{F}(t) \mathrm{d} t+\widetilde{f}(x), \quad \widetilde{F}\left(x_{0}\right)=X_{0}
$$

where $\lambda>0, a$ and $b$ are constants, $k(x, t)$ is an arbitrary kernel function and is continuous over the square $a \leq x, t \leq b$ and $f(x)$ is a function of $a \leq x \leq b$. Here we utilize the parametric forms of fuzzy functions.

\section{Homotopy Perturbation Method}

To illustrate the basic idea of homotopy perturbation method (see [8]), we consider the following nonlinear integral equation

$$
A(u)-f(r)=0, \quad r \in \Omega,
$$

with boundary conditions $B\left(u, \frac{\partial u}{\partial n}\right)=0, r \in \Gamma$, where $A$ is an integral operator, $B$ is a boundary operator, $\Omega$ is the boundary of the domain $\Omega$ and $f(r)$ is a 
known analytic function. Generally speaking, the operator $A$ can be divided into two parts $L$ and $N$, where $L$ is a linear and $N$ is a nonlinear operator. equation (2) can be rewritten as $L(u)+N(U)-f(r)=0$.

In order to use the HPM, a suitable construction of homotopy is of vital importance. If $L(u)=0$ with some possible unknown parameter can best describe the original nonlinear system. In general, a homotopy can be constructed as (see [5], [6])

$$
H(U, p)=(1-p)\left[L(U)-L\left(u_{0}\right)\right]+p[L(U)+N(U)-f(r)]=0
$$

or equivalently

$$
H(U, p)=L(U)-L\left(u_{0}\right)+p\left[L\left(u_{0}\right)+N(U)-f(r)\right]=0,
$$

where $u_{0}$ is an initial approximation of equation (2), $p[0,1]$ and $r \in \Omega$. It is obvious that

$$
H(U, 0)=L(U)-L\left(u_{0}\right)=0, \quad H(U, 1)=A(u)-f(r)
$$

and this deformation is called homotopy in topology. In this method, using the homotopy parameter $p$, we have the following power series representation for $U$, which is the solution of equation (2) or equation (4) where $0 \leq p \leq 1$

$$
u=u_{0}+p^{1} u_{1}+p^{2} u_{2}+\ldots
$$

The approximation can be obtained by setting $\mathrm{p}=1$, i.e.

$$
U=\lim _{p \rightarrow 1} u=\sum_{n=0}^{\infty} u_{n}=u_{0}+u_{1}+u_{2}+\ldots
$$

The above series is convergent for most cases, see [6].

\section{Proposed Algorithm for Solving Fuzzy Integro-Differential Equations}

One of our main aims is to present an efficient method to solve linear fuzzy integro-differential equation ( equation 1) and can be treated as an extended form of homotopy perturbation method, in which we use the accelerating parameter to enhance the rate of convergence of HPM applied to linear fuzzy integral equations of the second kind with the kernels of the form $\sum_{i=0}^{N} g_{i}(x) h_{i}(t)$. Then define new convex homotopy perturbation as follows

$$
H(u, p, m)=(1-p) F(u)+p L(u)+p(1-p)\left[\sum_{i=0}^{N} m_{i} g_{i}(x)\right]=0
$$


where $m=\left[m_{i}\right]$ and, $i=1,2,3, \ldots, N$ are called accelerating parameter for $m_{i}=0$. Now we define

$$
H(u, p, 0)=H(u, p),
$$

which is the standard HPM. It is easy to verify that the solutions of $H\left(u_{0}, 0\right)$ and $H(\gamma, 1)$ also satisfy the equation (8). To achieve our goal, we consider the linear fuzzy Fredholm integro-differential equation of the second kind as given in equation (1) with the solution that $\widetilde{F}(x)=\widetilde{u}(x)$, As the parametric forms of fuzzy functions are considered, we have $f(x)=(\underline{f}(x, \alpha), \bar{f}(x, \alpha))$.

Particular case: Consider the particular case, in which kernel function is of the form

$$
(\underline{k}(x, t), \bar{k}(x, t))=(\underline{g}(x) \underline{h}(t), \bar{g}(x) \bar{h}(t))
$$

We consider equation (8) as follows

$$
H(u, p, m)=\left\{\begin{array}{c}
(1-p) \underline{F} u+p L(u)+p(1-p)[\underline{m} \underline{g}(x)]=0 \\
(1-p) \bar{F} u+p L(u)+p(1-p)[\overline{m g}(x)]=0
\end{array}\right.
$$

where

$$
\begin{gathered}
(\underline{F}(u), \bar{F}(u))=\left\{\begin{array}{l}
\underline{u}^{\prime}(x, \alpha)-\underline{q}(x, \alpha) \underline{u}(x, \alpha)-\underline{f}(x, \alpha), \\
\bar{u}^{\prime}(x, \alpha)-\bar{q}(x, \alpha) \bar{u}(x, \alpha)-\bar{f}(x, \alpha),
\end{array}\right. \\
(\underline{L}(u), \bar{L}(u))= \\
\left\{\begin{array}{l}
\underline{u}^{\prime}(x, \alpha)-\underline{q}(x, \alpha) \underline{u}(x, \alpha)-\underline{f}(x, \alpha)-\int_{a}^{b} \underline{g}(x) \underline{h}(t) \underline{u}(t, \alpha) \mathrm{d} t=0 \\
\bar{u}^{\prime}(x, \alpha)-\bar{q}(x, \alpha) \bar{u}(x, \alpha)-\bar{f}(x, \alpha)-\int_{a}^{b} \bar{g}(x) \bar{h}(t) \bar{u}(t, \alpha) \mathrm{d} t=0
\end{array}\right.
\end{gathered}
$$

Hence we can write

$$
\begin{array}{r}
(1-p)\left(\underline{u}^{\prime}(x, \alpha)-\underline{q}(x, \alpha) \underline{u}(x, \alpha)-\underline{f}(x, \alpha)\right)+p\left(\underline{u}^{\prime}(x, \alpha)-\underline{q}(x, \alpha) \underline{u}(x, \alpha)\right. \\
\left.-\int_{a}^{b} \underline{g}(x) \underline{h}(t) \underline{u}(t, \alpha) \mathrm{d} t-\underline{f}(x, \alpha)\right)+\underline{m} p(1-p) \underline{g}(x)=0 \\
(1-p)\left(\bar{u}^{\prime}(x, \alpha)-\bar{q}(x, \alpha) \bar{u}(x, \alpha)-\bar{f}(x, \alpha)\right)+p\left(\bar{u}^{\prime}(x, \alpha)-\bar{q}(x, \alpha) \bar{u}(x, \alpha)\right. \\
\left.-\int_{a}^{b} \bar{g}(x) \bar{h}(t) \bar{u}(t, \alpha) \mathrm{d} t-\bar{f}(x, \alpha)\right)+\bar{m} p(1-p) \bar{g}(x)=0
\end{array}
$$


or equivalently

$$
\begin{aligned}
\underline{u}^{\prime}(x, \alpha) & -\underline{q}(x, \alpha) \underline{u}(x, \alpha)-\underline{f}(x, \alpha)+p \underline{g}(x) \int_{a}^{b} \underline{h}(t) \underline{u}(t, \alpha) \mathrm{d} t \\
& +\underline{m p} \underline{g}(x)+\underline{m}^{2} p \underline{g}(x)=0 \\
\bar{u}^{\prime}(x, \alpha)- & \bar{q}(x, \alpha) \bar{u}(x, \alpha)-\bar{f}(x, \alpha)+p \bar{g}(x) \int_{a}^{b} \bar{h}(t) \bar{u}(t, \alpha) \mathrm{d} t \\
& +\bar{m} p \bar{g}(x)+\bar{m}^{2} p \bar{g}(x)=0
\end{aligned}
$$

Substitute equation (6) in equation (15) and comparing the terms with like powers of $\mathrm{p}$ and we obtain

$$
p^{0}:= \begin{cases}\underline{u}_{0}^{\prime}(x, \alpha)-\underline{q}(x, \alpha) \underline{u}_{0}(x, \alpha)-\underline{f}(x, \alpha)=0, & \underline{u}_{0}(0)=\underline{X}_{0}(0) \\ \bar{u}_{0}^{\prime}(x, \alpha)-\bar{q}(x, \alpha) \bar{u}_{0}(x, \alpha)-\bar{f}(x, \alpha)=0, & \bar{u}_{0}(0)=\bar{X}_{0}(0)\end{cases}
$$

and its solution is

$$
\begin{aligned}
& \left\{\begin{array}{l}
\underline{u}_{0}(x, \alpha)=e^{\int_{a}^{b} \underline{q}(y, \alpha) \mathrm{d} y} \int_{a}^{x} e^{-\int_{a}^{t} \underline{q}(y, \alpha) \mathrm{d} y} \underline{f}(t, \alpha) \mathrm{d} t \\
\bar{u}_{0}(x, \alpha)=e^{\int_{a}^{b} \bar{q}(y, \alpha) \mathrm{d} y} \int_{a}^{x} e^{-\int_{a}^{t} \bar{q}(y, \alpha) \mathrm{d} y} \bar{f}(t, \alpha) \mathrm{d} t
\end{array}\right. \\
& p^{1}:\left\{\begin{array}{r}
\underline{u}_{1}^{\prime}(x, \alpha)=\underline{q}(x, \alpha) \underline{u}_{1}(x, \alpha)+\underline{g}(x) \int_{a}^{b} \underline{h}(t) \underline{u}_{0}(t, \alpha) \mathrm{d} t-\underline{m} \underline{g}(x), \\
\underline{u}_{1}(0)=0 \\
\bar{u}_{1}^{\prime}(x, \alpha)=\bar{q}(x, \alpha) \bar{u}_{1}(x, \alpha)+\bar{g}(x) \int_{a}^{b} \bar{h}(t) \bar{u}_{0}(t, \alpha) \mathrm{d} t-\bar{m} \underline{g}(x)=0, \\
\bar{u}_{1}(0)
\end{array}\right.
\end{aligned}
$$

Assume

$$
(\underline{c}, \bar{c})=\left\{\begin{array}{l}
\int_{a}^{b} \underline{h}(t) \underline{u}_{0}(t, \alpha) \mathrm{d} t \\
\int_{a}^{b} \bar{h}(t) \bar{u}_{0}(t, \alpha) \mathrm{d} t
\end{array}\right.
$$

which results in

$$
\left\{\begin{array}{l}
\underline{u}_{1}(x, \alpha)=(\underline{c}-\underline{m}) e^{\int_{a}^{x} \underline{q}(y, \alpha) \mathrm{d} y} \int_{a}^{x} e^{-\int_{a}^{t} \underline{q}(y, \alpha) \mathrm{d} y} \underline{g}(t) \mathrm{d} t \\
\bar{u}_{1}(x, \alpha)=(\bar{c}-\bar{m}) e^{\int_{a}^{x} \bar{q}(y, \alpha) \mathrm{d} y} \int_{a}^{x} e^{-\int_{a}^{t} \bar{q}(y, \alpha) \mathrm{d} y} \bar{g}(t) \mathrm{d} t
\end{array}\right.
$$




$$
p^{2}:\left\{\begin{array}{r}
\underline{u}_{2}^{\prime}(x, \alpha)=\underline{q}(x, \alpha) \underline{u}_{2}(x, \alpha)+\underline{g}(x) \int_{a}^{b} \underline{h}(t) \underline{u}_{1}(t, \alpha) \mathrm{d} t+\underline{m} \underline{g}(x), \\
\underline{u}_{2}(0)=0 \\
\bar{u}_{2}^{\prime}(x, \alpha)=\bar{q}(x, \alpha) \bar{u}_{2}(x, \alpha)+\bar{g}(x) \int_{a}^{b} \bar{h}(t) \bar{u}_{1}(t, \alpha) \mathrm{d} t+\overline{m g}(x), \\
\bar{u}_{2}(0)=0
\end{array}\right.
$$

Assume

$$
(\underline{s}, \bar{s})=\left\{\begin{array}{l}
\int_{a}^{b} \underline{h}(t) e^{\int_{a}^{t} \underline{q}(y, \alpha) \mathrm{d} y}\left(\int_{a}^{t} e^{-\int_{a}^{z} \underline{q}(y, \alpha) \mathrm{d} y} \underline{g}(z) \mathrm{d} z\right) \mathrm{d} t \\
\int_{a}^{b} \bar{h}(t) e^{\int_{a}^{t} \bar{q}(y, \alpha) \mathrm{d} y}\left(\int_{a}^{t} e^{-\int_{a}^{z} \bar{q}(y, \alpha) \mathrm{d} y} \bar{g}(z) \mathrm{d} z\right) \mathrm{d} t
\end{array}\right.
$$

Hence, we obtain

$$
\begin{aligned}
& \left\{\begin{array}{l}
\underline{u}_{2}(x, \alpha)=(\underline{m}+(\underline{c}-\underline{m}) \underline{s}) e^{\int_{a}^{x} \underline{q}(y, \alpha) \mathrm{d} y} \int_{a}^{x} e^{-\int_{a}^{t} \underline{q}(y, \alpha) \mathrm{d} y} \underline{g}(t) \mathrm{d} t \\
\left(\bar{u}_{2}(x, \alpha)=(\bar{m}+(\bar{c}-\bar{m}) \bar{s}) e^{\int_{a}^{x} \bar{q}(y, \alpha) \mathrm{d} y} \int_{a}^{x} e^{-\int_{a}^{t} \bar{q}(y, \alpha) \mathrm{d} y} \bar{g}(t) \mathrm{d} t\right.
\end{array}\right. \\
& p^{3}:\left\{\begin{array}{r}
\underline{u}_{3}^{\prime}(x, \alpha)-\underline{q}(x, \alpha) \underline{u}_{3}(x, \alpha)-\underline{g}(x) \int_{a}^{b} \underline{h}(t) \underline{u}_{2}(t, \alpha) \mathrm{d} t=0, \\
\underline{u}_{3}(0)=0 \\
\bar{u}_{3}^{\prime}(x, \alpha)-\bar{q}(x, \alpha) \bar{u}_{3}(x, \alpha)-\bar{g}(x) \int_{a}^{b} \bar{h}(t) \bar{u}_{2}(t, \alpha) \mathrm{d} t=0, \\
\underline{u}_{3}(0)=0
\end{array}\right.
\end{aligned}
$$

In general

$$
p^{n}:\left\{\begin{array}{r}
\underline{u}_{n}^{\prime}(x, \alpha)-\underline{q}(x, \alpha) \underline{u}_{n}(x, \alpha)-\underline{g}(x) \int_{a}^{b} \underline{h}(t) \underline{u}_{n-1}(t, \alpha) \mathrm{d} t=0, \\
\underline{u}_{n}(0)=0 \\
\bar{u}_{n}^{\prime}(x, \alpha)-\bar{q}(x, \alpha) \bar{u}_{n}(x, \alpha)-\bar{g}(x) \int_{a}^{b} \bar{h}(t) \bar{u}_{n-1}(t, \alpha) \mathrm{d} t=0, \\
\underline{u}_{n}(0)=0,
\end{array}\right.
$$

where $n=3,4,5, \ldots$ and it results in

$$
\left\{\begin{array}{l}
\underline{u}_{n}(x, \alpha)=\int_{a}^{b} \underline{h}(t) \underline{u}_{n-1}(t, \alpha) \mathrm{d} t e^{-\int_{a}^{x} \underline{q}(y, \alpha) \mathrm{d} y}\left(\int_{a}^{x} e^{-\int_{a}^{t} \underline{q}(y, \alpha) \mathrm{d} y} \underline{g}(t) \mathrm{d} t\right) \\
\bar{u}_{n}(x, \alpha)=\int_{a}^{b} \bar{h}(t) \bar{u}_{n-1}(t, \alpha) \mathrm{d} t e^{-\int_{a}^{x} \bar{q}(y, \alpha) \mathrm{d} y}\left(\int_{a}^{x} e^{-\int_{a}^{t} \bar{q}(y, \alpha) \mathrm{d} y} \bar{g}(t) \mathrm{d} t\right)
\end{array}\right.
$$


Now we will find the parameters $(\underline{m}, \bar{m})$ such that $\left(\underline{u}_{2}, \bar{u}_{2}\right)=0$,. Then it is obvious that $\left(\underline{u}_{3}, \bar{u}_{3}\right)=\left(\underline{u}_{4}, \bar{u}_{4}\right)=\ldots=0$. Hence the exact solution is obtained in the form

$$
(\underline{u}(x, \alpha), \bar{u}(x, \alpha))=\left(\underline{u}_{0}(x, \alpha)+\underline{u}_{1}(x, \alpha), \bar{u}_{0}(x, \alpha)+\bar{u}_{1}(x, \alpha)\right)
$$

Therefore, for all values of $x$, we have

$$
\left\{\begin{array}{l}
(\underline{m}+(\underline{c}-\underline{m}) \underline{s})=0 \\
(\bar{m}+(\bar{c}-\bar{m}) \bar{s})=0
\end{array}\right.
$$

or equivalently

$$
\begin{aligned}
& (\underline{m}, \bar{m})=\left(\frac{\underline{c s}}{\underline{s}-1}, \frac{\overline{c s}}{\bar{s}-1}\right) \\
& =\left\{\begin{array}{l}
\frac{\left(\int_{a}^{b} \underline{h}(t) \underline{u}_{0}(t, \alpha) \mathrm{d} t\right)\left(\int_{a}^{b} \underline{h}(t) e^{\int_{a}^{t} \underline{q}(y, \alpha) \mathrm{d} y}\left(\int_{a}^{t} e^{-\int_{a}^{t} \underline{q}(y, \alpha) \mathrm{d} y} \underline{g}(z) \mathrm{d} z\right) \mathrm{d} t\right)}{\int_{a}^{b} \underline{h}(t) e^{\int_{a}^{t} \underline{\underline{q}}(y, \alpha) \mathrm{d} y}\left(\int_{a}^{t} e^{-\int_{a}^{t} \underline{q}(y, \alpha) \mathrm{d} y} \underline{g}(z) \mathrm{d} z\right) \mathrm{d} t-1} \\
\frac{\left(\int_{a}^{b} \bar{h}(t) \bar{u}_{0}(t, \alpha) \mathrm{d} t\right)\left(\int_{a}^{b} \bar{h}(t) e^{\int_{a}^{t} \bar{q}(y, \alpha) \mathrm{d} y}\left(\int_{a}^{t} e^{-\int_{a}^{t} \bar{q}(y, \alpha) \mathrm{d} y} \bar{g}(z) \mathrm{d} z\right) \mathrm{d} t\right)}{\int_{a}^{b} \bar{h}(t) e^{\int_{a}^{t} \bar{q}(y, \alpha) \mathrm{d} y}\left(\int_{a}^{t} e^{-\int_{a}^{t} \overline{\bar{q}}(y, \alpha) \mathrm{d} y} \bar{g}(z) \mathrm{d} z\right) \mathrm{d} t-1}
\end{array}\right.
\end{aligned}
$$

with the condition that

$$
\left\{\begin{array}{l}
\left(\int_{a}^{b} \underline{h}(t) e^{\int_{a}^{t} \underline{q}(y, \alpha) \mathrm{d} y}\left(\int_{a}^{t} e^{-\int_{a}^{t} \underline{q}(y, \alpha) \mathrm{d} y} \underline{g}(z) \mathrm{d} z\right) \mathrm{d} t\right) \neq 1 \\
\left(\int_{a}^{b} \bar{h}(t) e^{\int_{a}^{t} \bar{q}(y, \alpha) \mathrm{d} y}\left(\int_{a}^{t} e^{-\int_{a}^{t} \bar{q}(y, \alpha) \mathrm{d} y} \bar{g}(z) \mathrm{d} z\right) \mathrm{d} t\right) \neq 1
\end{array}\right.
$$

General case: Let us consider the general case in which kernel is of the form

$$
(\underline{k}(x, t), \bar{k}(x, t))=\left(\sum_{i=1}^{N} \underline{g}_{i}(x), \underline{h}_{i}(t), \sum_{i=1}^{N} \bar{g}_{i}(x), \bar{h}_{i}(t)\right)
$$

In this case, we choose the convex homotopy as

$$
H(u, p, m)=\left\{\begin{array}{l}
(1-p) \underline{F} u+p L(u)+p(1-p)\left(\sum_{i=1}^{N} \underline{m}_{i} \underline{g}_{i}(x)\right)=0 \\
(1-p) \bar{F} u+p L(u)+p(1-p)\left(\sum_{i=1}^{N} \bar{m}_{i} \bar{g}_{i}(x)\right)=0
\end{array}\right.
$$


On using similar computations, we obtain the following results in which equation (3) and equation (13) is same in this case also, whereas we have

$p^{1}:\left\{\begin{array}{l}\underline{u}_{1}^{\prime}(x, \alpha)-\underline{q}(x, \alpha) \underline{u}_{1}(x, \alpha)-\sum_{i=1}^{N}\left(\int_{a}^{b} \underline{h}_{i}(t) \underline{u}_{0}(t, \alpha) \mathrm{d} t-\underline{m}_{i}\right) \underline{g}_{i}(x), \\ \underline{u}_{1}(0)=0 \\ \bar{u}_{1}^{\prime}(x, \alpha)-\bar{q}(x, \alpha) \bar{u}_{1}(x, \alpha)-\sum_{i=1}^{N}\left(\int_{a}^{b} \bar{h}_{i}(t) \bar{u}_{0}(t, \alpha) \mathrm{d} t-\bar{m}_{i}\right) \bar{g}_{i}(x), \\ \bar{u}_{1}(0)=0\end{array}\right.$

$$
p^{2}:\left\{\begin{array}{l}
\underline{u}_{2}^{\prime}(x, \alpha)-\underline{q}(x, \alpha) \underline{u}_{2}(x, \alpha)=\sum_{i=1}^{N}\left(\underline{h}_{i}(t) \underline{u}_{1}(t, \alpha) \mathrm{d} t+\underline{m}_{i}\right) \underline{g}_{i}(x), \\
\underline{u}_{2}(0)=0 \\
\bar{u}_{2}^{\prime}(x, \alpha)-\bar{q}(x, \alpha) \bar{u}_{2}(x, \alpha)=\sum_{i=1}^{N}\left(\bar{h}_{i}(t) \bar{u}_{1}(t, \alpha) \mathrm{d} t+\bar{m}_{i}\right) \bar{g}_{i}(x), \\
\bar{u}_{2}(0)=0
\end{array}\right.
$$

Comparing the co-responding powers of $p$, we have simliar results for $p^{3}$ also. Hence we have the following general form

$p^{n}:\left\{\begin{array}{l}\underline{u}_{n}(x, \alpha)=\int_{a}^{b} \underline{h}_{i}(t) \underline{u}_{n-1}(t, \alpha) \mathrm{d} t e^{\int_{a}^{x} \underline{q}(y, \alpha) \mathrm{d} y}\left(\int_{a}^{x} e^{-\int_{a}^{t} \underline{q}(y, \alpha) \mathrm{d} y} \underline{g}_{i}(t) \mathrm{d} t\right) \\ \bar{u}_{n}(x, \alpha)=\int_{a}^{b} \bar{h}_{i}(t) \bar{u}_{n-1}(t, \alpha) \mathrm{d} t e^{\int_{a}^{x} \bar{q}(y, \alpha) \mathrm{d} y}\left(\int_{a}^{x} e^{-\int_{a}^{t} \bar{q}(y, \alpha) \mathrm{d} y} \bar{g}_{i}(t) \mathrm{d} t\right)\end{array}\right.$

where $n=3,4,5, \ldots$ and now we find the parameters $\left(\underline{m}_{i}, \bar{m}_{i}\right), i=1,2,3, \ldots, N$ such that $\left(\underline{u}_{2}, \bar{u}_{2}\right)=0$, it is obvious $\left(\underline{u}_{3}, \bar{u}_{3}\right)=\left(\underline{u}_{4}, \bar{u}_{4}\right)=\ldots=0$. Hence from equation (40) for every $x \epsilon[a, b]$,

$$
\left\{\begin{array}{c}
\int_{a}^{b} \underline{h}_{i}(t) \underline{u}_{1}(t, \alpha) \mathrm{d} t+\underline{m}_{i}=0 \\
\int_{a}^{b} \bar{h}_{i}(t) \bar{u}_{1}(t, \alpha) \mathrm{d} t+\bar{m}_{i}=0
\end{array}\right.
$$


Substitute equation (34) in equation (36), we receive

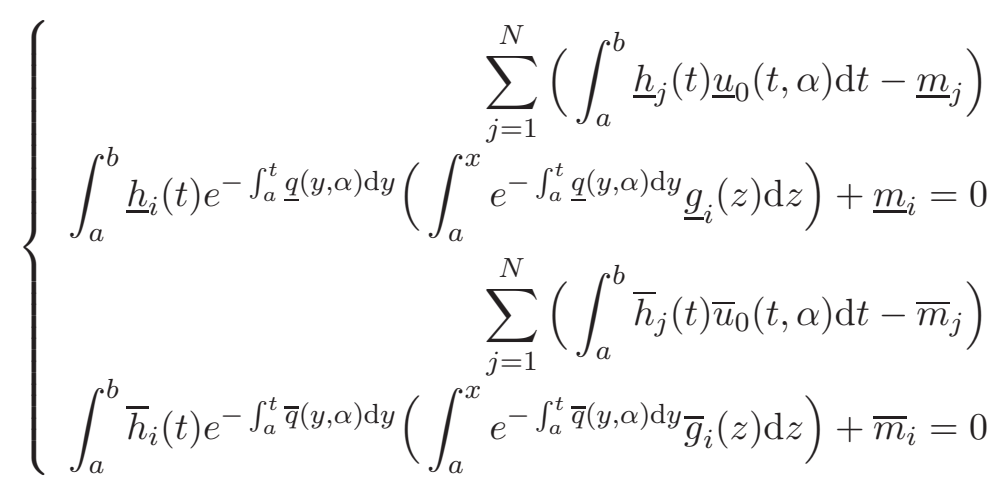

Let us set

$$
\begin{gathered}
\left\{\begin{array}{l}
\underline{c}_{j}=\int_{a}^{b} \underline{h}_{j}(t) \underline{u}_{0}(t, \alpha) \mathrm{d} t \\
\bar{c}_{j}=\int_{a}^{b} \bar{h}_{j}(t) \bar{u}_{0}(t, \alpha) \mathrm{d} t
\end{array}\right. \\
\left\{\begin{array}{l}
\underline{b}_{j}=\int_{a}^{b} \underline{h}_{i}(t) e^{\int_{a}^{t} \underline{q}(y, \alpha) \mathrm{d} y}\left(\int_{a}^{t} e^{\left.-\int_{a}^{z} \underline{q}^{(y, \alpha) \mathrm{d} y} \underline{g}_{j}(z) \mathrm{d} z\right)}\right. \\
\bar{b}_{j}=\int_{a}^{b} \bar{h}_{i}(t) e^{\int_{a}^{t} \bar{q}(y, \alpha) \mathrm{d} y}\left(\int_{a}^{t} e^{-\int_{a}^{z} \bar{q}(y, \alpha) \mathrm{d} y} \bar{g}_{j}(z) \mathrm{d} z\right)
\end{array}\right.
\end{gathered}
$$

Then, we recceive

$$
\left\{\begin{array}{l}
\sum_{j=1}^{N} \underline{b}_{i j}\left(\underline{c}_{j}-\underline{m}_{j}\right)+\underline{m}_{i}=0 \\
\sum_{j=1}^{N} \bar{b}_{i j}\left(\bar{c}_{j}-\bar{m}_{j}\right)+\bar{m}_{i}=0
\end{array}\right.
$$

The value of the accelerating parameter $\left(\underline{m}_{i}, \bar{m}_{i}\right), i=1,2,3, \ldots, N$ can be found under certain condition, from the system of linear equations in equation (47). Let us assume the matrix as $(\underline{A}, \bar{A})=\left(\underline{b}_{i j}, \bar{b}_{i j}\right)$ and the vectors as $(\underline{m}, \bar{m})=$ $\left(\underline{m}_{j}, \bar{m}_{j}\right)$ and $(\underline{c}, \bar{c})=\left(\underline{c}_{j}, \bar{c}_{j}\right)$. Thus from equation $(47)$, we can write $((\underline{A}-$ $\underline{I}) \underline{m},(\bar{A}-\bar{I}) \bar{m})=(\underline{A c}, \bar{A} \bar{c})$, where $(\underline{I}, \bar{I})$ is the identity matrix and suppose if we have $((\underline{A}-\underline{I}),(\bar{A}-\bar{I}))$ is non-singular, then we have $(\underline{m}, \bar{m})=\left((\underline{A}-\underline{I})^{-1} \underline{A c},(\bar{A}-\right.$ $\left.\bar{I})^{-1} \bar{A} \bar{c}\right)$ which clearly gives all the values of $\left(\underline{m}_{i}, \bar{m}_{i}\right), i=1,2,3, \ldots, N$. 


\section{Conclusion}

In this paper, we developed an efficient and appropriate method to solve fuzzy integro-differential equations. Reliability of HPM due to its precise results and reduction in computation gives it a wider applicability. Proposed method based on HPM presented in the form of convergent series with easily computable components avoids tedious work needed by the traditional methods. Advantage of the proposed method lies in the free selection of initial approximation in a straightforward manner and also it overcomes the drawbacks of handling larger equations. Since there are challenging issues to solve these equations, our method is trustworthy and we showed that construction of proper homotopy leads to highly accurate solutions and is considered as a remarkable benefit of this algorithm undoubtedly.

\section{References}

[1] T. Allahviranloo, S. Abbasbandy and S. Hashemzehi, Approximating the solution of the linear and nonlinear fuzzy Volterra integrodifferential equations using expansion method, Abstract and Applied Analysis, Article ID 713892, 2014 (2014), 1-7.

[2] D. Dubois and H. Prade, Towards fuzzy differential calculus, Fuzzy Sets and Systems, 8 (1982), 1-7.

[3] O. Ghasemi, M. Tavassoli Kajani and E. Babolian, Application of He's homotopy perturbation method to nonlinear integro-differential equations, Applied Mathematics and Computation, 188 (2007), 538-548.

[4] R. Goetschel, W. Voxman, Elementary fuzzy calculus, Fuzzy Sets and Systems, 18 (1986), 31-43.

[5] J. H. He, Homotopy perturbation technique, Comput Methods Appl Mech Engrg, 178 (1999), 257-262.

[6] J. H. He, A coupling method of a homotopy technique and a perturbation technique for non-linear problems, International Journal of Non-Linear Mechanics, 35 (2000), 37-43.

[7] O. Kaleva, Fuzzy differential equations, Fuzzy Sets and Systems, 24, No. 2 (1987), 301317.

[8] S. Narayanamoorthy and S. P. Sathiyapriya, Homotopy perturbation method: a versatile tool to evaluate linear and nonlinear fuzzy Volterra integral equations of the second kind, SpringerPlus, 5, 387 (2016), 1-16, doi: 10.1186/s40064-016-2038-3.

[9] S. Narayanamoorthy and S. P. Sathiyapriya, A pertinent approach to solve nonlinear fuzzy integro-differential equations, SpringerPlus, 5:449 (2016), 1-17, doi: 10. $1186 / \mathrm{s} 4006401620454$.

[10] J. Saberi-Nadjafi and A. Ghorbani, Hes homotopy perturbation method: An effective tool for solving integral and integro-differential equations, Computers and Mathematics with Appications, 58 (2009), 2379-2390.

[11] L. A. Zadeh, Fuzzy Sets, Information and Control, 8 (1965), 338-353. 